\title{
Early interfaced neural activity from chronic amputated nerves
}

\author{
Kshitija Garde ${ }^{1}$, Edward Keefer', Barry Botterman'2, Pedro Galvan ${ }^{3}$ and Mario I. Romero ${ }^{1,3 *}$ \\ 1 Department of Plastic Surgery, University of Texas Southwestern Medical Center, Dallas, TX, USA \\ 2 Department of Cell Biology, University of Texas Southwestern Medical Center, Dallas, TX, USA \\ ${ }^{3}$ Regenerative Neurobiology Research Division, Texas Scottish Rite Hospital for Children, Dallas, TX, USA
}

Edited by:

Stefano Ferraina, Sapienza University

of Rome, Italy

Reviewed by:

Thomas Phely-Bobin, Materials

Technology Foster-Miller, Inc., USA

Xavier Navarro, Universitat Autonoma

de Barcelona, Spain

Yael Hanein, Tel Aviv University, Israel

${ }^{*}$ Correspondence:

Mario I. Romero-Ortega, The University

of Texas at Arlington, Bioengineering

Department, 501 W. First Street,

ELB Room 220, Box 19138, Arlington,

TX 76019, USA.

e-mail:mromero@uta.edu

\begin{abstract}
Direct interfacing of transected peripheral nerves with advanced robotic prosthetic devices has been proposed as a strategy for achieving natural motor control and sensory perception of such bionic substitutes, thus fully functionally replacing missing limbs in amputees. Multielectrode arrays placed in the brain and peripheral nerves have been used successfully to convey neural control of prosthetic devices to the user. However, reactive gliosis, micro hemorrhages, axonopathy and excessive inflammation currently limit their long-term use. Here we demonstrate that enticement of peripheral nerve regeneration through a non-obstructive multi-electrode array, after either acute or chronic nerve amputation, offers a viable alternative to obtain early neural recordings and to enhance long-term interfacing of nerve activity. Non-restrictive electrode arrays placed in the path of regenerating nerve fibers allowed the recording of action potentials as early as 8 days post-implantation with high signal-to-noise ratio, as long as 3 months in some animals, and with minimal inflammation at the nerve tissue-metal electrode interface. Our findings suggest that regenerative multi-electrode arrays of open design allow early and stable interfacing of neural activity from amputated peripheral nerves and might contribute towards conveying full neural control and sensory feedback to users of robotic prosthetic devices.
\end{abstract}

Keywords: nerve injury, nerve regeneration, neural interface, multi-electrode array, neuroprostheses

\section{INTRODUCTION}

Over 1.7 million people suffer from limb loss in the United States and this number is estimated to increase by 185,000 a year for upper extremity loss alone (Owings and Kozak, 1998). Interfacing advanced robotic prosthetic devices with the nervous system of amputees offers the possibility of functionally replacing their missing limbs by enabling them to naturally control and feel a bionic substitute. Robotic limb replacement for chronic amputees has been recently achieved using electromyographic signals obtained from reinervated targeted chest muscles to control the robotic prosthesis (Hijjawi et al., 2006; Miller et al., 2008).

This approach successfully provides gross voluntary control of the prosthetic limb, but offers limited incorporation of sensory feedback to the nerve needed for precise control and natural sensation of the artificial limb. Regenerated arm sensation overlaps with chest sensations, and when elicited by either touch or electrical stimulation, conveys referred sensations of large areas or multiple disparate sensations across the hand (Kuiken et al., 2007). This limitation obligates the users to rely on visual feedback to move and position their prosthetic limbs, which places a cognitive burden on the use of such devices. Recent compelling evidence has demonstrated that voluntary movement of robotic prosthetic limbs can be achieved with dexterity by extracting planned movement information from the motor cerebral cortex or the peripheral nerve via multi-electrode array neurointerfaces (Hochberg et al., 2006; Normann, 2007). Moreover, sensory feedback can also be directly conveyed to amputees via electrical microstimulation of the sensory cortex (Fitzsimmons et al., 2007) or peripheral nerves (Dhillon and Horch, 2005; Normann, 2007).
Different types of multi-electrode arrays have been developed to obtain high spatial and temporal resolution sampling of motor neuronal activity (reviewed by Cheung, 2007). Unfortunately, irrespective of the probe design, current electrodes provide limited long-term efficacy due to progressive reduction in the number of active sites over time. This reduction is presumably a result of poor tissue-electrode interface, tissue damage by probe micromotion within the soft nerve tissue, and electrode insulation due to tissue scar formation (Biran et al., 2005, 2007; Leung et al., 2008; Williams et al., 2007). These limitations obligate the need for neurointerface recalibration prior to each recording session and gradual increase in the voltage needed to maintain neural responses over time, which in turn raise the risk of electrolytic tissue damage and further compromise the long-term stability of the neurointerface.

Regenerative sieve electrodes were proposed more than three decades ago as a viable alternative to interface motor and sensory nerve fibers (Dario et al., 1998; Edell et al., 1982; Mannard et al., 1974), and have been shown to be useful in obtaining neural recordings after long-term (i.e., 2-6 months) implantation (Klinge et al., 2001; Lago et al., 2007; Panetsos et al., 2008). Unfortunately, neural activity seems to be obtained only from a fraction of the animals tested, and only a low proportion of the electrodes on the sieve seem to provide recordable signals (Lago et al., 2005). Furthermore, the initial functional recovery reported in implanted animals worsens over time, which seems to be caused by compressive axonopathy due to mechanical injury and the inability of the ring-electrode design to accommodate the normal increase in axon size during maturation and re-myelination (Castro et al., 2008; Lago et al., 2007). 
The present study tested the hypothesis that multi-electrode arrays placed in the path of regenerating nerve fibers with minimal or no restriction to the regenerative process, would provide improved neural interfaces to the amputated peripheral nervous system. We demonstrate that non-obstructive regenerative neurointerfaces can be used to successfully obtain early neural recordings and to enhance long-term electrophysiological recordings from acutely or chronically amputated nerves.

\section{MATERIALS AND METHODS MULTI-ELECTRODE ARRAY NERVE GUIDE}

Custom-made floating arrays with 18-pin Parylene-C insulated platinum/iridium electrodes were used (150-250 kohm impedance at $1 \mathrm{kHz}$, Microprobes Inc, MD, USA). Individual electrodes were approximately $50 \mu \mathrm{m}$ in diameter at the shank, varied in height (0.5-0.9 mm with $2 \mu \mathrm{m}$ active tips), and were placed $400 \mu \mathrm{m}$ apart to maximize contact with the regenerating nerve fibers. The array cable was fabricated from Palylene-C insulated $25 \mu \mathrm{m}$ gold wires wound in helix and coated with MDX4-4210 silicone elastomer and was $17 \mathrm{~cm}$ in length. Microarrays were placed in the lumen of polyurethane nerve guide tubes prior to implantation (MicroRenathane $^{\circledR}$, Braintree Scientific, Inc; OD $3 \mathrm{~mm}$, ID $1.75 \mathrm{~mm}$, and $7 \mathrm{~mm}$ in length). The entire assembly was sterilized and the lumen filled with collagen I/III (0.3\% Chemicon, Tamacula, CA, USA) prior to surgery (Figure 1A).

\section{ANIMAL SURGERY}

Eighteen adult Lewis rats (150-250 kg), divided in four groups, were used in this study: Non-injured controls (I; $n=4$ ), acutely implanted animals after sciatic nerve injury with either a simple tube (II; $n=4$ ) or the electrode array nerve guide (III; $n=5$ ), and animals implanted with the electrode nerve guide after chronic sciatic nerve injury (IV; $n=5)$. The animals were anaesthetized (ketamine/medetomidine, $87 \mathrm{mg} / \mathrm{kg}$ i.p.) before being subjected to sciatic nerve transection injury. After acute (same day; Figure 1C) or chronic (180 days later; Figure 1D) nerve injury, the multielectrode nerve guide was implanted and sutured at proximal and distal ends leaving a nerve gap of approximately $5 \mathrm{~mm}$. The array was then connected to an 18-pin headcap (Omnetics Connection Co.) via subcutaneous gold microwires (Figure 1B). To model chronic nerve amputation and limb loss, the sciatic nerve that normally innervates the gastrocnemious, tibial, and common peroneal muscles was transected and sutured to the biceps femoris (abnormal target), 6 months prior to implantation of the neural interface. Animals were placed in cages with soft cellu-dri bedding and kept in a humidity/temperature controlled room with a 12-h light/dark cycle with food and water ad libitum. Animal care and surgical procedures were performed in accordance to the Institutional Animal Care and Use Committee at UT Southwestern Medical Center.

\section{ELECTROPHYSIOLOGY}

Neural activity was recorded weekly for $45 \mathrm{~min}$ in freely moving animals with a 16-channel recorder with the capacity to record from 64 neurons simultaneously (i.e. 4 per channel at $25 \mathrm{~s}$ precision and $40 \mathrm{kHz}$ resolution). The recorded signals were amplified and digitized by a multichannel acquisition processor (MAP; Plexon Inc, Dallas) according to published methodology (Nicolelis,
2003). Visual inspection of the analog signal on the oscilloscope and the digitized 16-channel MAPs were used to identify events above the noise level that were then recorded and further processed using a Data Offline Spike Sorter program (OFSS, Plexon) to isolate single units from the noise by 3-component Principal Component Analysis. All the channels had independent control over gain, notch filters and reference to maximize the extraction of information from the neuronal activity. Sorted waveforms were further analyzed using NeuroExplorer/Spike Histogram Software (Nex Technologies, Littelton, MA, USA) to evaluate changes in the pattern of firing (bursting or spiking) of the regenerated axons.

\section{BEHAVIORAL ANALYSIS}

Sensory and motor tests to ascertain functional recovery were performed as previously described (Romero et al., 2000). Briefly, nociceptive function was evaluated by eliciting foot withdrawal response after noxious heat stimulation using the Ugo Basile plantar device. The infrared thermal probe was positioned under the plantar surface of the paw to apply incremental heat to a maximum of $55^{\circ} \mathrm{C}$ for $20 \mathrm{~s}$. The paw withdraw latency was measured twice at 10-min intervals. A paw licking response elicited by the thermal stimulation was also scored as either present or absent. A toe spreading assay was also performed as reported to evaluate motor function recovery (Romero et al., 2007). The extent of toe spread was analyzed by measuring the distance between the first and fifth toes bilaterally, and the toe spread index estimated by calculating the ratio of injured-to-non-injured sides.

\section{HISTOLOGY}

Animals were euthanized at 21-240 days after implantation according to multi-electrode array function (median $=58$ days). The sciatic nerve was carefully dissected and fixed overnight in $4 \%$ paraformaldehyde. The multi-electrode array was then carefully removed, the proximal and distal ends of the nerve were labeled, and the tissue processed for paraffin embedding. Serial $4 \mu \mathrm{m} \mathrm{sec}-$ tions were obtained and immunostained for specific markers for Schwann cells (polyclonal anti-S100 1:300; DAKO Z0311), neurons (mouse anti-200 KD Neurofilament Protein 1:300; Dako M0762, rabbit anti- $\beta$-tubulin 1:500 and anti-calcitonin gene-related peptide 1:1500; Chemicon Int.), activated macrophages (mouse anti-ED1 1:50; Chemicon Int.) and myelin (anti-MBP; 1:50; Chemicon Int.). Tissue sections from all experimental groups were processed simultaneously using identical incubating solutions. Immunodetection of these markers was visualized by DAB used as an HRP substrate. Mayers-Hematoxylin treatment followed by ammonia water was used as a counter-stain. For immunofluorsecence studies, the deparaffinized sections were blocked with $5 \%$ normal goat serum incubated overnight with the primary antibodies, and reacted with Cy2 Goat anti-Rabbit 1:250, Cy3 and Cy2 Goat anti-Mouse 1:500; Cy3 Goat anti-Rat 1:400 (Jackson labs) for visualization. Sections were mounted using Vectashield containing the nuclear label DAPI (Molecular Probes).

\section{DIGITAL IMAGE ANALYSIS}

The proximity of regenerating axons to the surface electrode was evaluated from NFP-stained sections at 40× magnification using light microscopy. The distance from the electrode shaft and nearest 


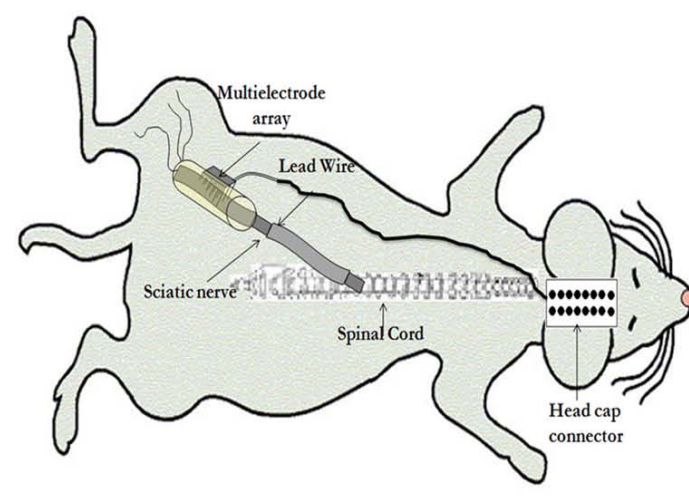

A
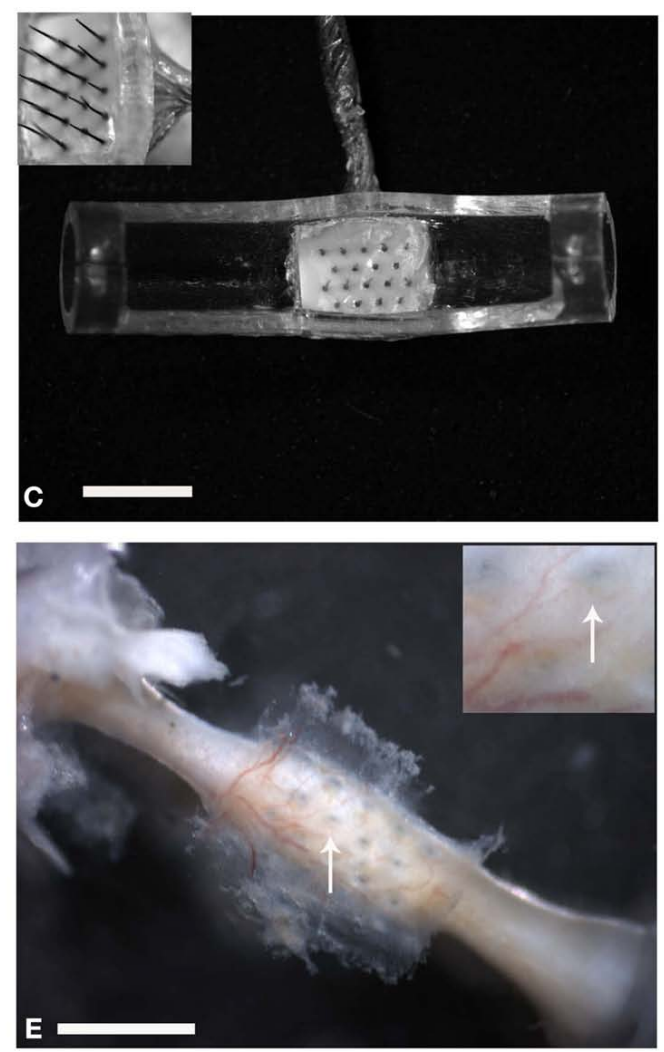
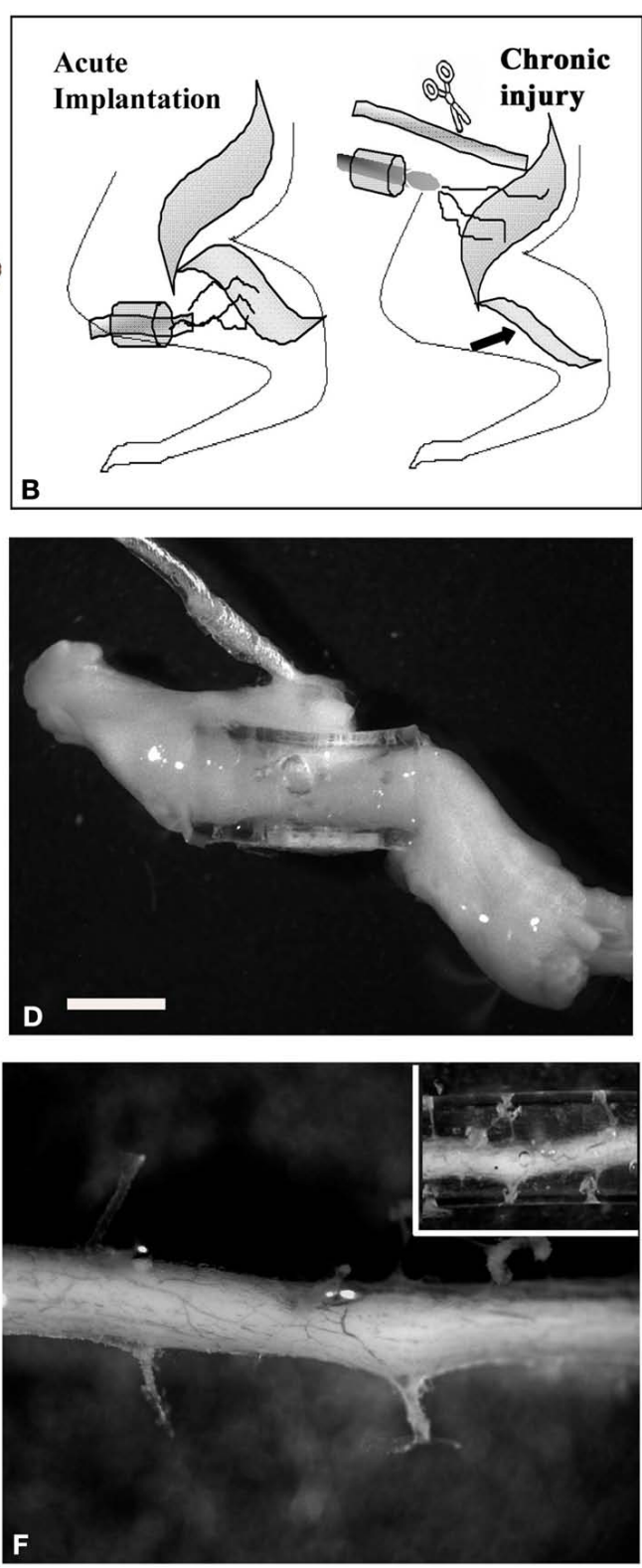

FIGURE 1 | Multi-electrode array nerve implants. (A) Illustration of the electrode array nerve guide and head cap connection. Schematic representation of the acute and chronic injury/implantation paradigm [(B) arrow indicates denervation induced atrophy of the normal target muscle]. (C) Top view of the 18 pin multi-electrode array mounted in a tubular nerve guide (top removed for clarity). The length of the electrodes varied from $0.5-0.9 \mathrm{~mm}$, with the taller ones placed in the center of the array (insert). (D) Photograph of the regenerated nerve through the electrode-conduit assembly in an acute animal 3 weeks post-implantation. (E) Photograph showing the perforations left by the MEA in a chronic injured implanted animal (arrows in insert) and (F) through a collagen-filled tube. Scale bar $=2 \mathrm{~mm}$ (C), $5 \mathrm{~mm}$ (D) and $800 \mu \mathrm{m}$ (E). axon per electrode was measured using the Axiovision analysis software (Axiovision Inc). The extent of inflammation near the electrodes was also determined by measuring the length of ED1 immunoreactivity in four different quadrants $\left(x-x^{\prime} ; y-y^{\prime}\right)$ around the circular area of each electrode as visualized from the stained horizontal sections. Data was obtained from 5-17 electrodes per animal. To determine the extent of nerve regeneration through the electrode nerve guide, the regenerated nerve obtained from animals with acute implantation of the array were immulolabeled for $\beta$-tubulin and measured in triplicate for optical densitometry in areas approximately $1 \mathrm{~mm}$ proximal and distal from the array.

\section{STATISTICAL ANALYSIS}

All data were reported as the mean and the standard error of the mean (SEM). An unpaired Student's $t$-test was used to determine statistical differences. In multiple group comparisons, one-way 
ANOVA was used followed by Neuman-Keuls multiple comparison post hoc evaluation (Prism 4, GraphPad). p-Values $\leq 0.05$ were considered significant.

\section{RESULTS}

Nerve regeneration was observed in all animals along the multielectrode nerve guide; both after acute implantation or after grafting onto chronically amputated nerves. In most animals (8/10) the regenerated tissue covered the nerve guide lumen (Figure 1D), whereas in two animals the regenerated tissue was found partially covering the multi-electrode array (i.e., the tips of the larger electrodes were exposed as the regenerated nerve did not fill the lumen of the tube). Several weeks (4-12) after implantation, the entire nerve guide including the electrode base was found to be encapsulated by vascularized fibrous tissue. Dissection of the fibrotic tissue and removal of the array revealed normal healing and tissue regeneration through the electrode array (Figure 1E), which was comparable with those animals with simple tubularization repairs (Figure 1F), indicating that the placement of the electrodes in the lumen of the nerve guide did not drastically hamper the nerve regenerative process.

To directly demonstrate axonal regeneration through the multi-electrode nerve guide, paraffin sections of the regenerated tissue at proximal, middle, and distal levels of the nerve guide were immunolabeled for the specific neuronal marker $\beta$-tubulin. Representative sections from animals either implanted at the time of injury (Figures 2A-C), or after chronic nerve amputation (Figures 2D-F) showed abundant axons of normal morphology circumventing the electrodes at the middle of the array and into the distal nerve stump, demonstrating that regenerating neurons successfully traversed the multi-electrode array placed in the lumen of the nerve guide. Quantification of nerve regeneration through the electrode nerve guide as determined by $\beta$-tubulin densitometry at the proximal and distal ends of the regenerate, indicated that $64.58 \pm 7.42 \%$ of the axons were able to transverse the electrode array in animals with acute nerve injuries. The cause of reduced nerve regeneration in the distal tissue of chronically amputated animals (Figure 2F) is unclear. However, it might reflect the reported diminished capacity of neurons to regenerate after chronic denervation (Fu and Gordon, 1995).

Regenerating axons visualized by immunodetection of the neurofilament protein (NFP) revealed nerve fiber growth in close proximity to the electrodes. On average, axons were found to be closer to the electrode shafts in the acute repaired animals $(12.13 \pm 3.03 \mu \mathrm{m})$, compared to those that received the implant after several weeks of nerve amputation $(28.28 \pm 12.18 \mu \mathrm{m})$. Axonal profiles were also found to be separated from the electrodes by a well organized three to eight cell layer (Figures 3A,B), suggesting some degree of tissue encapsulation of the foreign body.

We confirmed that Schwann cells were localized along the regenerated axons by the co-localization of $\beta$-tubulin with S-100 near the electrodes (Figures 3C,D). We then evaluated whether nerve regeneration across the multi-electrode nerve guide was successful for both small diameter unmyelinated fibers as well as large diameter myelinated axons. Tissue sections were stained for the visualization of a specific marker for myelinated axons; myelin basic protein (MBP; Figure 3E), and that of TrkA+ nociceptive pain fibers; calcitonin gene-related peptide (CGRP; Figure 3F). The results indicated that both types of neurons, likely sensory and motor axons, were able to regenerate in near proximity to the active recording sites of the array. Furthermore, visualization of nodes of Ranvier near the electrodes suggested normal myelination of the regenerated nerve fibers.
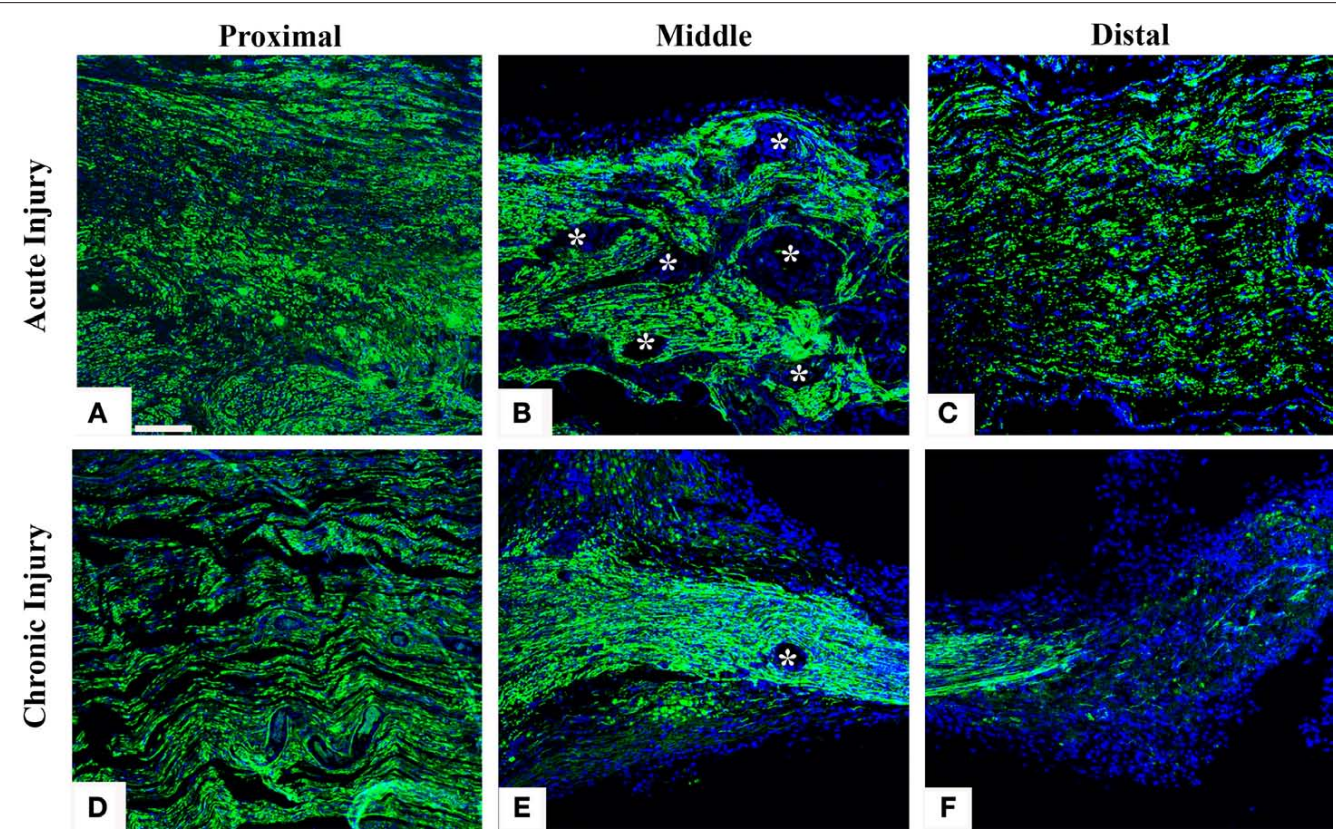

FIGURE 2 | Nerve regeneration through the multi-electrode array. Specific neuronal labeling by $\beta$-tubulin immunostaining (green) demonstrates axonal regeneration around the electrodes (asterisks) in both acute $(\mathbf{A}-\mathbf{C})$ and chronic (D-F) injured nerves. DAPI (blue) counter staining. Scale bar $=50 \mu m$. 

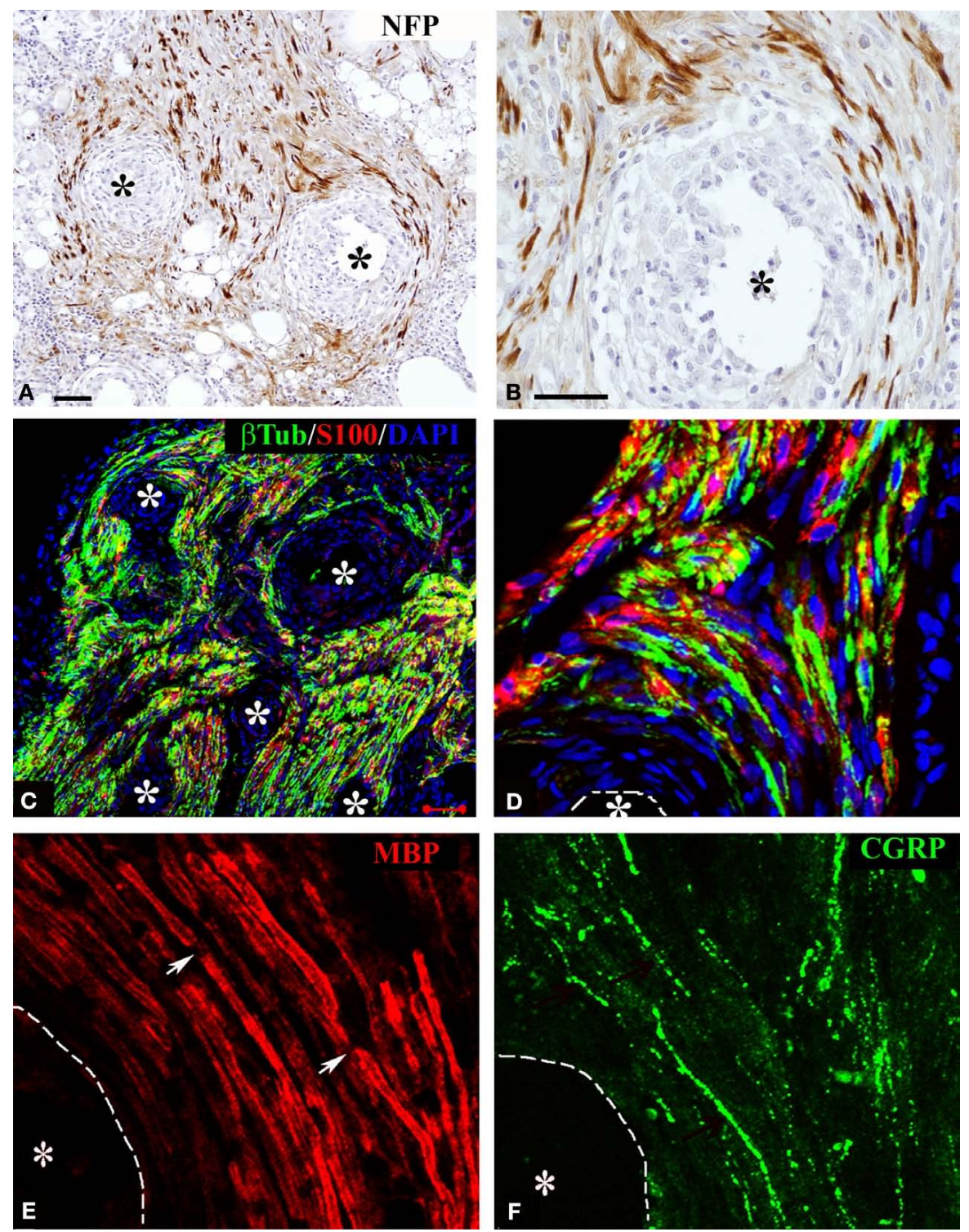

FIGURE 3 | Regeneration of myelinated and unmyelinated fibers.

Neurofilament immunostaining labeled axons regenerating around the shaft of the electrodes [asterisks (A,B)]. Schwann cells (S-100+) were visualized along regenerated axons [ $\beta$-tubulin+ $(\mathbf{C}, \mathbf{D})]$. Both myelinated [(E) MBP+; red] and unmyelinated (pain) fibers [(F) CGRP+; green] were visualized in close proximity to the electrodes (dotted line). Nodes of Ranvier were observed near the electrodes [arrows in (E)]. Scale bars $=50 \mu \mathrm{m}(\mathbf{A}, \mathbf{C})$ and $25 \mu \mathrm{m}$ (B,D-F)

\section{LIMITED MACROPHAGE ACTIVATION AT THE ELECTRODE-TISSUE INTERFACE}

The presence of cells at the tissue-electrode interface suggested some degree of inflammation. To evaluate such response we visualized activated macrophages by immunodetection of the specific ED-1 marker at both horizontal and coronal planes (Figure 4A). Qualitative analysis revealed a relatively low number of macrophages in the area directly in contact with the electrodes, which marked precisely the localization of the electrodes in the regenerated tissue (Figures $4 \mathrm{~B}, \mathrm{C}$ ). The accumulation of macrophages around the electrodes was consistent in all animals and limited to two to five cell layers at the bio-abio interface. Quantification of the inflammation area as determined by the length of ED-1 immunoreactivity from the electrode surface to the last positive cell in cross section, revealed a slightly but significantly larger inflammation around the multi-electrode array in chronically injured animals $(30 \pm 1 \mu \mathrm{m})$ compared to those implanted at the time of injury (20 $\pm 5 \mu \mathrm{m}$; Figure 4D).

To determine if the presence of activated macrophages affected axonal growth or re-myelination, we evaluated the co-localization of ED-1 with NFP and MBP, respectively. As shown in Figure 5, after acute (Figures 5A,B) or chronic (Figures 5C,D) injury to the nerve, regenerated axons were separated from the electrodes by a layer of macrophages. However, ED-1/MBP co-staining suggested 


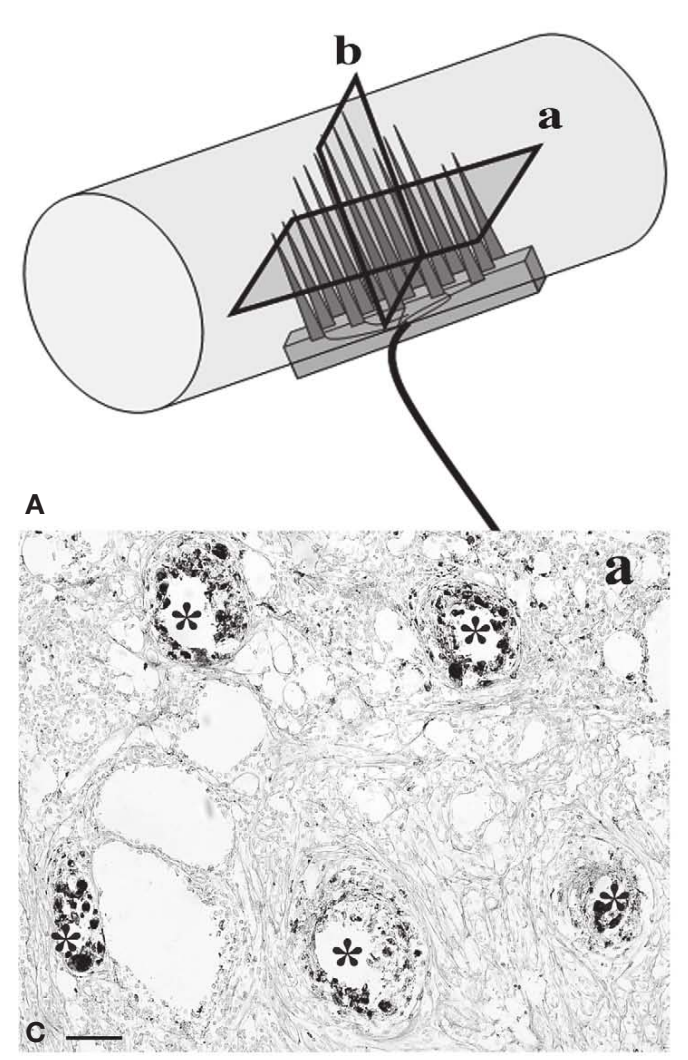

FIGURE 4 | Minimal inflammation elicited by the multi-electrode array (A) Schematic of the multi-electrode array mounted in the nerve guide showing the sectioning planes (a-horizontal; b-coronal) used for analysis. Representative coronal (B) and horizontal (C) tissue sections show highly localized ED-1
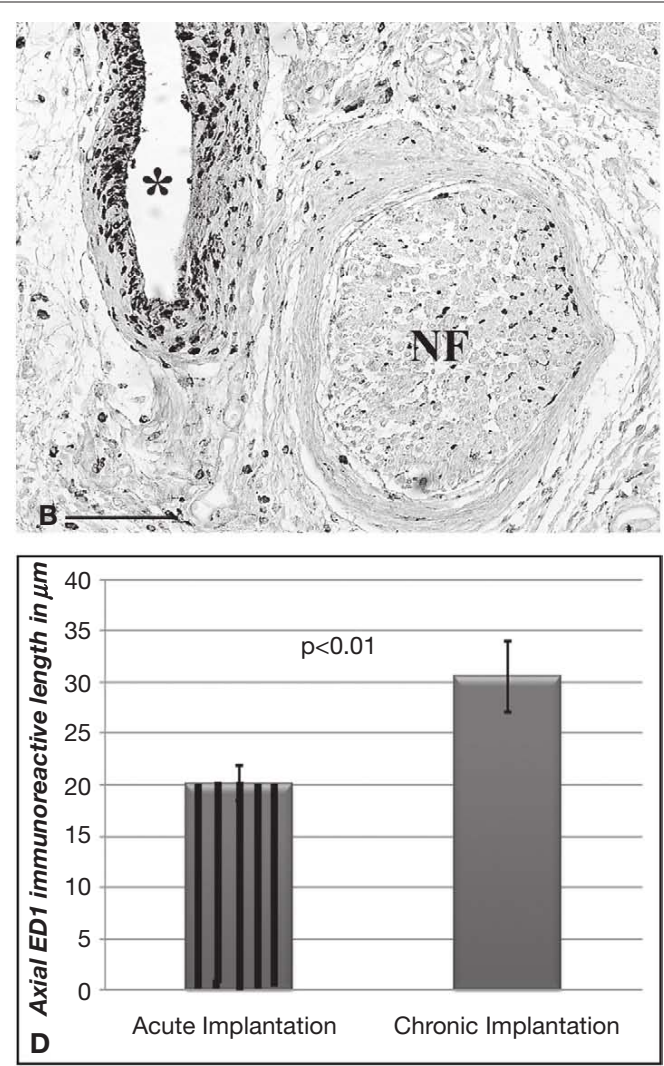

immunolabeling around the electrodes. (D) Quantification of ED-1+ cellular thickness in such areas revealed a slight but significant increase in the inflammatory response of animals with chronic nerve injuries compared to acute implanted animals. Scale bars $=100 \mu \mathrm{m}$ (B) and $50 \mu \mathrm{m}$ (C). NF $=$ nerve fascicle normal myelination of axons adjacent to the ED-1 positive cells (Figure 5B) as indicated by the normal nerve morphology despite the presence of the inflammatory cells. Together, the histological data demonstrate nerve regeneration across a guide deploying an array of 18 needle electrodes with no evidence of tissue damage or axonal compression, and with minimal inflammation at the tissue-electrode interface.

\section{REGENERATIVE NEUROINTERFACES REVEALED EARLY AND STABLE NEURAL ACTIVITY}

We successfully recorded from 7/10 animals and were able to detect neural activity at most of the active sites, in some cases for more than 3 months after implantation. Spontaneous neural activity from the regenerated peripheral neurons was obtained as early as 8 days post-implantation. A total of six units from four electrodes were recorded at this very early time of regeneration. The signal to noise ratio was estimated to be $6: 1$ with a mean background noise of $300 \mu \mathrm{V}$ and neuron action potential amplitudes ranging from 0.3 to $1 \mathrm{mV}$. Figure 6 shows examples of single (Figure 6A) and multiunit activity (Figure 6C) isolated either after acute injury (Figures 6A-D) or after 223 days of nerve amputation (Figures 6G,H). The earliest recording was obtained 1 week after implantation, where one of the animals showed single units with typical extracellular signal shapes in 5/16 electrodes, and multi-unit detection in two additional electrodes. Principal component analysis was used to identify distinct 3D spatial cluster distribution neural activity and confirm the presence of separate action potentials within a multiunit detection. As expected, neural activity was elicited upon voluntary movement or evoked by thermal or mechanical (i.e., Von Frey filament and joint stretch) sensory stimulation (Figures 6B,C). With time, an increasing trend in the number of active recording sites was apparent in most animals, likely reflecting an increasing number of regenerated axons. However, in some of the electrodes, the ability to record single unit spiking decreased after a few weeks (Figure 6G vs. Figure 6H).

\section{FUNCTIONAL RECOVERY IN END-TO-END NEUROINTERFACES}

The amount of nerve regeneration observed through the regenerative neurointerface suggested that the multi-electrode array might have allowed target reinnervation and functional recovery after implantation into the peripheral nerve. In order to confirm this possibility, we evaluated motor functional recovery in a cohort of animals in which the array was acutely implanted into short nerve gaps with preserved normal target innervations, and compared to those in which nerve reinnervaton was prevented. We evaluated the ability of the implanted animals to recover reflex-elicited toe abduction as estimated by the injured-to-non-injured toe spread index (TSI). Compared to normal animals in which toe extension 

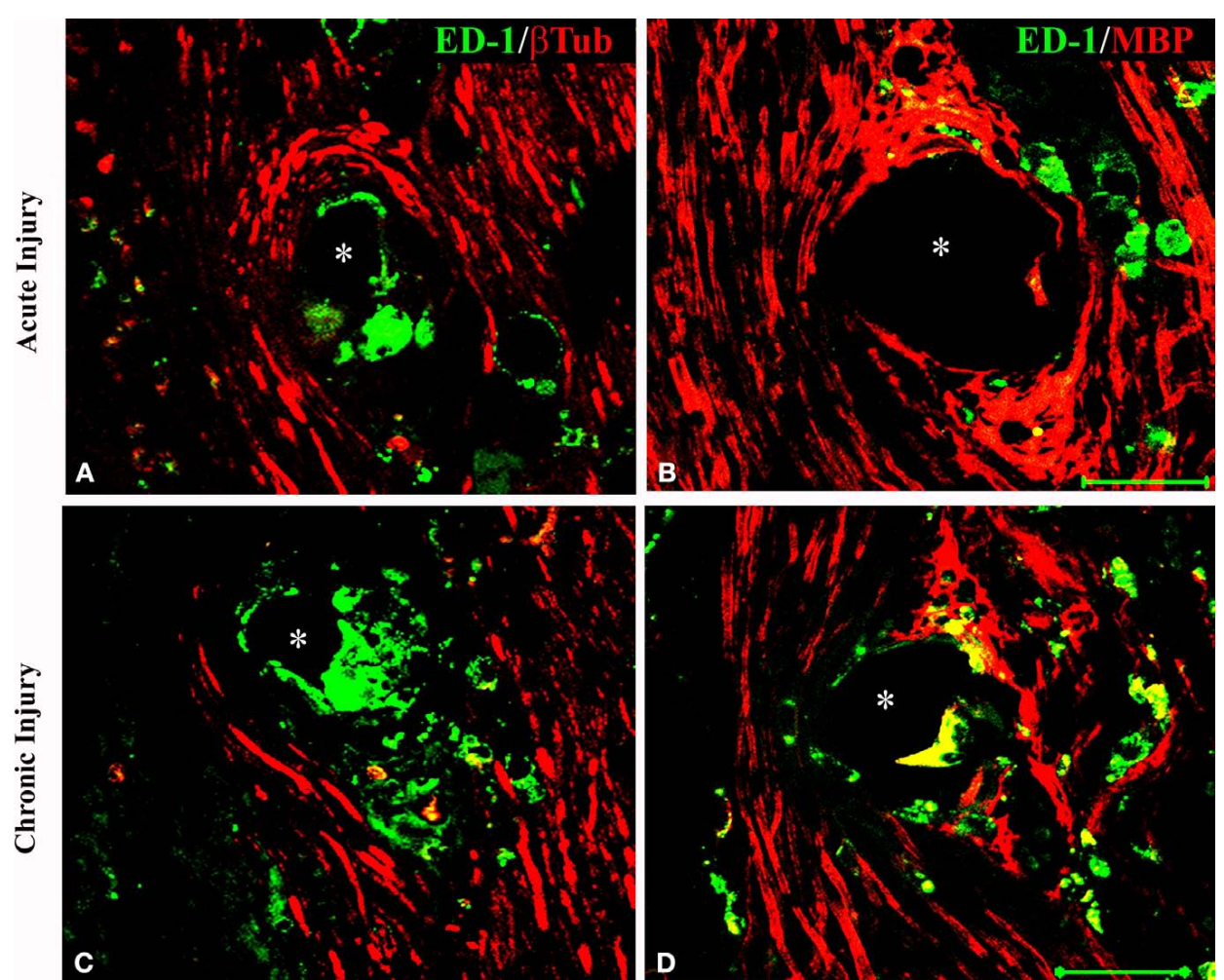

FIGURE 5 |Activated macrophages at the nerve-electrode interface. Animals implanted at the time of injury $(\mathbf{A}, \mathbf{B})$ or 6 months after nerve amputation (C,D), showed axonal regeneration and the immune response elicited by the electrodes as indicated by the co-labeling of axons $[\boldsymbol{\beta}$-tubulin $(\mathbf{A}, \mathbf{C})$ and $\mathrm{MBP}(\mathbf{B}, \mathbf{D})]$ and activated macrophages (ED-1+) at the tissue-electrode interface, both near the tip $(\mathbf{A}, \mathbf{C})$ or the base $\mathbf{( B , D )}$ of the electrode shaft. Scale bar $=50 \mu \mathrm{m}$. was similar in both paws of control animals (Figures 7A,D), injured animals in which nerve regeneration to their original target was prevented by connecting the transected nerve to an aberrant muscle (i.e., bicep femoris), showed "claw" toes and approximately 70\% reduction in toe extension (Figures 7 B,D). Conversely, animals in which the multi-electrode array was used to repair an acute gap injury with normal target innervations (i.e., gastrocnemious muscle) recovered their motor function as indicated by the significant improvement in toe spreading compared to denervated animals (Figures 7C,D; $p \leq 0.03$ ). This data correlated with the presence of acetylcholine receptors in the neuromuscular junctions of the gastrocnemius muscle (data not shown). In addition, pain perception in these animals was evaluated by eliciting a paw licking response after exposure of the plantar surface to a noxious thermal stimulus. As expected, injured animals in which normal target reinnervation was prevented showed no sensory response with a paw withdrawal latency (PWL) of $17.45 \pm 1.41 \mathrm{~s}$, compared to non-injured controls $(\mathrm{PWL}=10.23 \pm 0.97 \mathrm{~s})$. In contrast, animals with end-to-end repairs showed a licking behavior comparable to that observed in non-injured animals with PWL responses of $8.75 \pm 2.02 \mathrm{~s}$ in the implanted limb. The data is consistent with the notion that interfacing the peripheral nerve with non-obstructive multi-electrode arrays would allow them to reconnect with their original target organs and to mediate functional recovery. However, it does not completely rule out the possibility of a behavioral response elicited by stimulation of the saphenous nerve in some of the animals.

\section{DISCUSSION}

Neural control of robotic prosthesis has progressed dramatically in recent years with animal and clinical studies demonstrating the feasibility of voluntary motor neural control of artificial limbs (Hochberg et al., 2006; Lebedev and Nicolelis, 2006; Normann, 2007). However, developing a stable and long-lasting neurointerface that would allow for specific multichannel recording/stimulation remains to be achieved (Navarro et al., 2005). Edell et al. (1982) exploited the natural ability of peripheral nerves for spontaneous regeneration to develop a sieve multi-electrode array aimed at providing improved neurointerfacing. Unfortunately, only a small fraction of regenerating axons, mostly sensory in nature, appears to be able to grow through the sieve electrode. In addition, the long-term use of this neurointerface is limited due to compression axonopathy and distal degeneration (Castro et al., 2008).

Here we report that peripheral nerves, whether acutely injured or implanted after months of chronic amputation, can be interfaced early by enticing them to grow in close proximity to electrodes placed in a tridimensional open regenerative setting. This was demonstrated by the ability to record $300-1000 \mu \mathrm{V}$ action potentials as early as 8 days post-implantation in this study. The obtained recorded neural activity seemed larger and more readily available compared to those reported using sieve electrodes (i.e., 100-200 $\mu \mathrm{V}$ with early recordings obtained at 29 days postimplantation) (Mensinger et al., 2000). Action potential amplitudes, however, are known to vary greatly depending on the type 

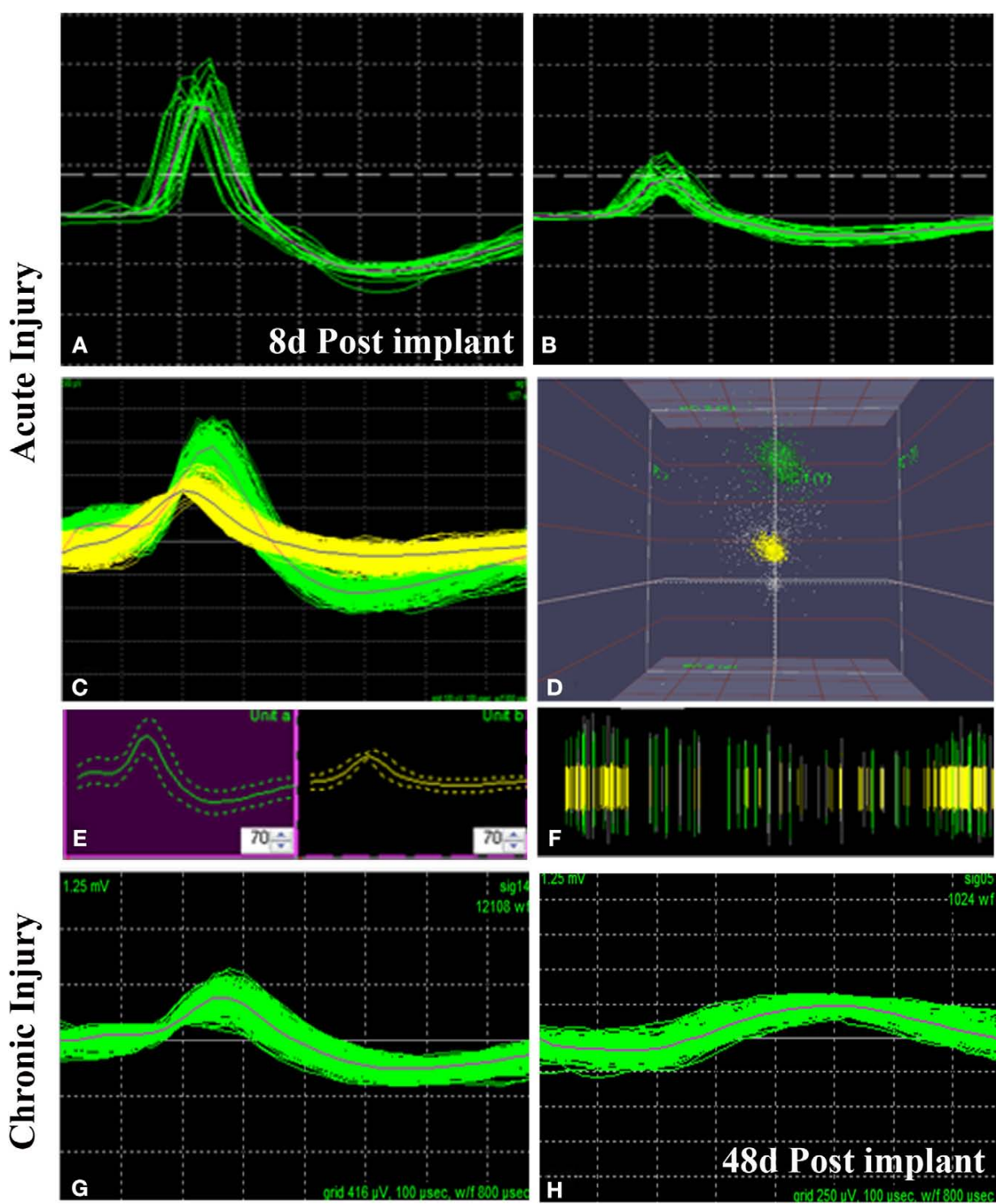

FIGURE 6 | Neural activity recorded from freely moving animals. Single $(\mathbf{A}, \mathbf{B})$ and multiple $\mathbf{( C )}$ spike activity could be detected in both acute (A-C) and chronic (G) injured animals as early as 8 days post-implantation. The amplitude of the neural spike varied in both types of animals [compared (A) vs. (B) and (B) vs. (G)]. Identification of separate units was confirmed by principal component analysis and 3-D cluster plot (C-E). Spike frequency is shown in (F). Overall, the amplitude of neural activity recorded at the same electrode at 8 days $(\mathbf{G})$ and 48 days $(\mathbf{H})$ of implantation showed attenuation of signal overtime. $\mathrm{X}$ scale $=100 \mu \mathrm{V}$ and Y scale $=100 \mu \mathrm{s}$ of electrodes and the recorded tissue. Michigan electrodes have recorded amplitudes ranging from $50-800 \mu \mathrm{V}$ in brain (Vetter et al., 2004). In the peripheral nerve the recorded action potentials range from $150-600 \mu \mathrm{V}$ in early reports of regenerative interfaces using the sieve electrode (Edell et al., 1982), 15-300 $\mu \mathrm{V}$ using the Utah multi-electrode arrays (Vetter et al., 2004), and 20-40 $\mu \mathrm{V}$ using intrafascicular electrodes (Dhillon et al., 2004).

We favor the interpretation that the increased signal amplitude in our study results from axons regenerating in close proximity to the electrodes, as it is well known that extracellular depolarization amplitude falls over the square of distance. This notion is supported by the following observations: First, the multi-electrode array did not seem to obstruct nerve regeneration as axons readily grew through the array circumventing the electrodes, and functional recovery was achieved if implants were connected distally to their appropriate target organs. Second, the number of active electrodes increased over time reaching stability in number within the first 3 weeks, correlating with the time needed for axonal regeneration through the nerve guide. Third, neural recordings were obtained as early as 1 week after implantation of the electrode array in animals with either acute injury or 5-6 months after nerve amputation. Fourth, minimal inflammation was observed at the neuron/electrode interface.

Despite such indications, the early neural signals recorded in this study progressively reduced in amplitude over time, suggesting 

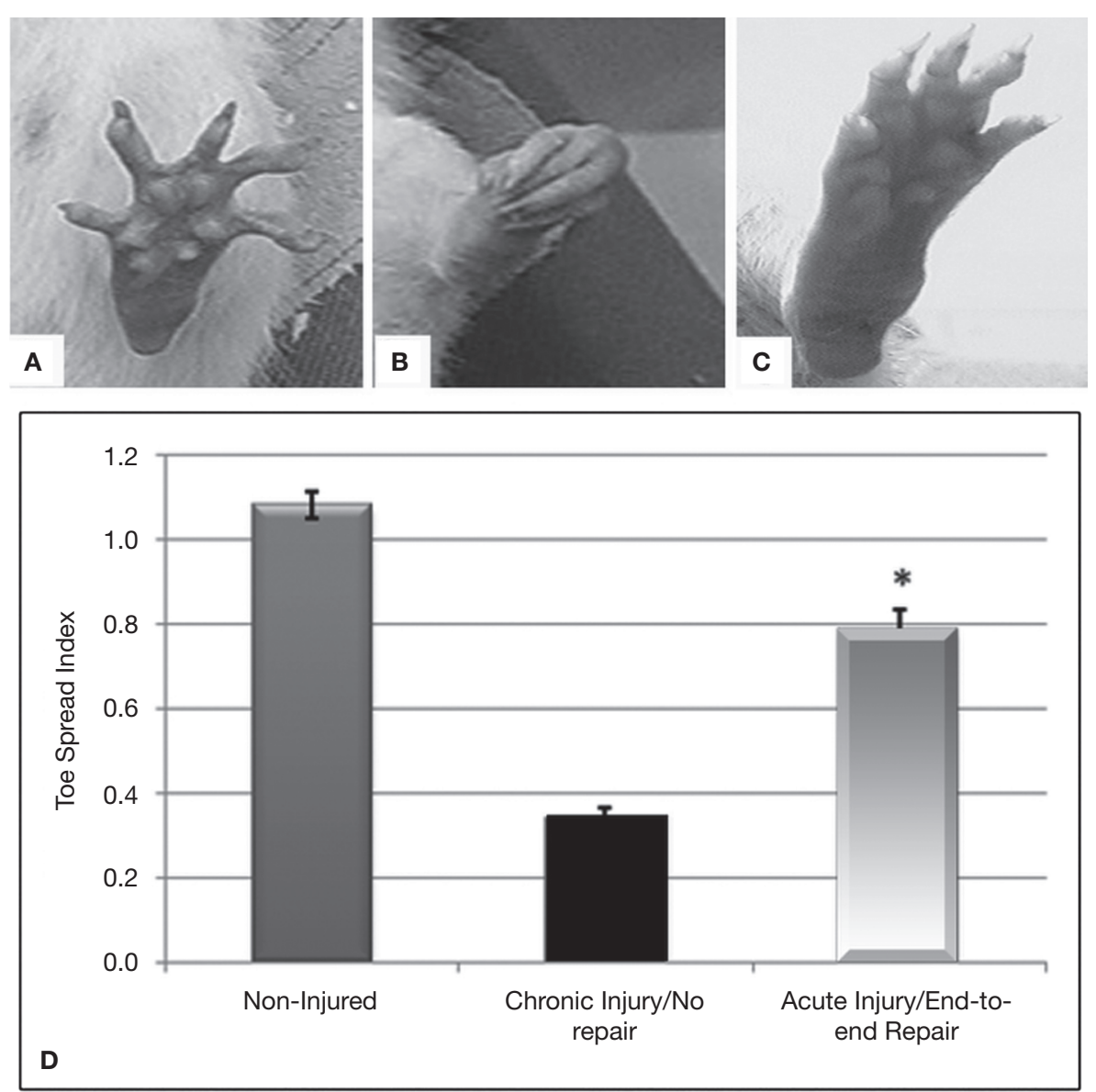

FIGURE 7 | Functional recovery in interfaced animals. The toe spread index (TSI) was evaluated to determine whether functional reinnervation occurred despite neural interfacing to the multi-electrode nerve guide. Compared to noninjured animals (A), chronically injured animals in which the nerve was attached to an abnormal target failed to recover (B). In contrast, animals with acute endto-end neurointerfaces allowed to reinnervate their original targets, showed significant functional recovery compared to chronically denervated animals (C-D). ${ }^{*} p \leq 0.5$. that factors such as electrode insulation by activated macrophages and myelination, need to be minimized in order to increase further the sensitivity and long-term stability of regenerative peripheral nerve interfaces.

\section{LIMITING THE FOREIGN BODY RESPONSE TO THE METAL ELECTRODES}

A discrete cell layer of activated macrophages was found adjacent to the metal electrodes in this study, extending over a 35 and $65 \mu \mathrm{m}$ radius in the acute and chronically injured animals, respectively. The observed inflammatory response elicited by the electrodes seems reduced compared to those in brain recordings using conventionally tethered silicon microarrays and in the peripheral nerve using chronically implanted indwelling multi-electrode arrays. In the brain, ED1+ reactive cells are known to extend over a 100-250 $\mu$ m radius from the electrodes (Biran et al., 2007) and in the PNS approximately $430 \mu \mathrm{m}$ of collagen are deposited at the base of the array and $34 \mu \mathrm{m}$ at the electrode shaft (Branner et al., 2004; Lago et al., 2007). The limited inflammation observed in the present study might be related to the formation of a nerve/electrode array monolithic structure resulting from the fibrotic encapsulation of the implant, which is less susceptible to use-related damage due to tethering forces and mechanical damage normally associated to all the indwelling electrodes (Polikov et al., 2005). Therefore, macrophage activation in the present study seems likely to be evoked primarily by a foreign body response at the bio-abio interface. Overcoming this limitation will likely require the incorporation of strategies aimed at increasing the biocompatibility of the metal electrodes. In that line of thought, it has been recently reported that coating metal electrodes with the extracellular matrix protein laminin, significantly reduces macrophage activation in rat brain tissue (He et al., 2006). Thus, similar strategies might offer a viable alternative to further reduce the inflammation elicited by the multi-electrode array in the transected peripheral nerve. While standard immunossupresion techniques can be incorporated to minimize the immunological response to the electrodes (Kim and Martin, 2006; Polikov et al., 2005), they are also likely to interfere with the regenerative process.

\section{ALTERNATIVE REGENERATIVE ELECTRODE ARRAY DESIGNS}

Here we demonstrate that electrode arrays which do not restrict the growth of the regenerating nerve fibers offer a viable 
alternative for neurointerfacing amputated peripheral nerves. Although we used needle-shape multi-electrodes in this study, tissue penetration is obviously not required during nerve regeneration. Therefore, alternative electrode designs such as fibers, pillars, cylinders, semicircles, or spirals, might represent a better alternative for developing more sensitive regenerative neurointerfaces as they might better incorporate the necessary characteristics to amplify the amplitude of the recorded nerve signals as predicted by mathematical simulation studies (Fitzgerald et al., 2008; Loeb et al., 1977).

Both sensitivity and long-term stability of the neurointerface can potentially be further enhanced by the incorporation of neurotrophic factors (Rejali et al., 2007; Seymour and Kipke, 2007; Yamagata et al., 2004), and coating the metal electrode with conductive polymers (Ludwig et al., 2006; Richardson et al., 2007) or nanomaterials (Wang et al., 2006; Yu et al., 2007). We and others have demonstrated that pristine (Galvan-Garcia et al., 2007) or chemically modified (Mattson et al., 2000; Ni et al., 2005) carbon nanotubes (CNTs) can serve as enticing material for neural growth and as viable substrates for neuron recording and stimulation in vitro (Lovat et al., 2005; Mazzatenta et al., 2007). Recently, Keefer et al. (2008) have demonstrated that electroplating platinum electrodes with CNTs-gold or CNTs-polypyrrole significantly enhances the electrical characteristics of the electrodes by decreasing the impedance and increasing the sensitivity and charge storage capacity. Thus, it is likely that future electrode designs that incorporate biological and nanomaterial components will provide improved electrical properties in capacitance, and signal-to-noise ratio, which will further improve the sensitivity and long-term stability of either peripheral or central neural interfaces.

\section{REFERENCES}

Biran, R., Martin, D. C., and Tresco, P. A. (2005). Neuronal cell loss accompanies the brain tissue response to chronically implanted silicon microelectrode arrays. Exp. Neurol. 195, 115-126.

Biran, R., Martin, D. C., and Tresco, P. A. (2007). The brain tissue response to implanted silicon microelectrode arrays is increased when the device is tethered to the skull. J. Biomed. Mater. Res. A 82, 169-178.

Branner, A., Stein, R. B., Fernandez, E., Aoyagi, Y., and Normann, R.A. (2004). Long-term stimulation and recording with a penetrating microelectrode array in cat sciatic nerve. IEEE Trans. Biomed. Eng. 51, 146-157.

Castro, J., Negredo, P., and Avendano, C. (2008). Fiber composition of the rat sciatic nerve and its modification during regeneration through a sieve electrode. Brain Res. 1190, 65-77.

Cheung, K. C. (2007). Implantable microscale neural interfaces. Biomed. Microdevices 9, 923-938.

Clements, I. P., Young-Tae, K., Andreasen, D., and Bellamkonda, R. V. (2007). A regenerative electrode scaffold for peripheral nerve interfacing. In CNE'07.3rdInternational IEEE/EMBS

As with other reports using wired peripheral neurointerfaces, most failures in this study were found to be due to breaks in the wire either at the junction with the multi-electrode array or at the head connector. Moreover, the subcutaneous wiring and head cap were areas commonly associated with irritation and infection, which also limited the survival times for some animals in this study. The development of polyimide thin-film electrode arrays (Clements et al., 2007) and wireless neurointerfaces bear great promise in obviating some of these limitations and allowing the stable recording/stimulation of long-term preparations (Ghovanloo and Najafi, 2007).

In summary, our findings indicate that non-obstructive regenerative multi-electrode arrays can guide nerve regeneration in close proximity to their active recording sites, eliciting minimal inflammation and providing early and relatively stable neurointerfacing to both acute and chronic injured peripheral nerves. Such properties might be advantageous in providing neural control and functional feedback information to users of robotic prosthetic devices. In addition, functional target reinnervation in end-to-end acute neurointerfacing suggests that regenerative electrode arrays might be useful for applications in which chronic neurostimulation and preservation of the normal target innervation (i.e., pain management and bladder control restoration) is desired.

\section{ACKNOWLEDGEMENTS}

This work was supported by Defense Advanced Research Projects Agency and the Crowely-Carter Foundation (MIR). We thank Russ Daniel for administrative assistance, Myresa Hurst for expert technical support, and members of the Romero' lab for comments on the manuscript.

Fitzsimmons, N.A., Drake, W.,Hanson, T. L. Lebedev, M. A., and Nicolelis, M.A. (2007). Primate reaching cued by multichannel spatiotemporal cortical microstimulation. J. Neurosci. 27, 5593-5602.

Fu, S. Y., and Gordon, T. (1995). Contributing factors to poor functional recovery after delayed nerve repair: prolonged denervation. J. Neurosci. 15(Pt 2), 3886-3895.

Galvan-Garcia, P., Keefer, E. W., Yang, F., Zhang, M., Fang, S., Zakhidov, A. A., Baughman, R. H., and Romero, M. I. (2007). Robust cell migration and neuronal growth on pristine carbon nanotube sheets and yarns. J. Biomater. Sci. Polym. Ed. 18, 1245-1261.

Ghovanloo, M., and Najafi, K. (2007). A wireless implantable multichannel microstimulating system-on-a-chip with modular architecture. IEEE Trans. Neural Syst. Rehabil. Eng. 15, 449-457.

He, W., McConnell, G. C., and Bellamkonda, R. V. (2006). Nanoscale laminin coating modulates cortical scarring response around implanted silicon microelectrode arrays. J. Neural Eng. 3, 316-326.

Hijjawi,J.B., Kuiken, T.A.,Lipschutz, R. D., Miller, L. A., Stubblefield, K. A., and Dumanian, G. A. (2006). Improved myoelectric prosthesis control accomplished using multiple nerve transfers. Plast. Reconstr. Surg. 118, 1573-1578.

Hochberg, L. R., Serruya, M. D., Friehs, G. M., Mukand, J.A., Saleh, M. Caplan, A. H., Branner, A., Chen, D., Penn, R. D., and Donoghue, J. P. (2006). Neuronal ensemble control of prosthetic devices by a human with tetraplegia. Nature 442, 164-171.

Keefer, E. W., Botterman, B. R., Romero, M. I., Rossi, A. F., and Gross, G. W. (2008). Carbon nanotube coating improves neuronal recordings. Nat. Nanotechnol. 3, 434-439.

Kim, D. H., and Martin, D. C. (2006) Sustained release of dexamethasone from hydrophilic matrices using PLGA nanoparticles for neural drug delivery. Biomaterials 27, 3031-3037.

Klinge, P. M., Vafa, M. A., Brinker, T., Brandis, A., Walter, G. F., Stieglitz, T. Samii, M., and Wewetzer, K. (2001). Immunohistochemical characterization of axonal sprouting and reactive tissue changes after long-term implantation of a polyimide sieve electrode to the transected adult rat sciatic nerve. Biomaterials 22, 2333-2343.

Kuiken, T. A., Marasco, P. D., Lock, B. A., Harden, R. N., and Dewald, J.P. (2007). 
Redirection of cutaneous sensation from the hand to the chest skin of human amputees with targeted reinnervation. Proc. Natl. Acad. Sci. U.S.A. 104, 20061-20066.

Lago, N., Ceballos, D., Rodriguez, F. J., Stieglitz, T., and Navarro, X. (2005). Long term assessment of axonal regeneration through polyimide regenerative electrodes to interface the peripheral nerve. Biomaterials 26, 2021-2031.

Lago,N.,Udina,E., Ramachandran,A., and Navarro, X. (2007). Neurobiological assessment of regenerative electrodes for bidirectional interfacing injured peripheral nerves. IEEE Trans. Biomed. Eng. 54(Pt 1), 1129-1137.

Lebedev, M. A., and Nicolelis, M. A. (2006). Brain-machine interfaces: past, present and future. Trends Neurosci. 29, 536-546.

Leung, B. K., Biran, R., Underwood, C. J., and Tresco,P.A.(2008).Characterization of microglial attachment and cytokine release on biomaterials of differing surface chemistry. Biomaterials 29, 3289-3297.

Loeb, G. E., Marks, W. B., and Beatty, P. G. (1977). Analysis and microelectronic design of tubular electrode arrays intended for chronic, multiple singleunit recording from captured nerve fibres. Med. Biol. Eng. Comput. 15, 195-201.

Lovat, V., Pantarotto, D., Lagostena, L., Cacciari, B., Grandolfo, M., Righi, M., Spalluto, G., Prato, M., and Ballerini, L. (2005). Carbon nanotube substrates boost neuronal electrical signaling. Nano Lett. 5, 1107-1110.

Ludwig, K. A., Uram, J. D., Yang, J., Martin, D. C., and Kipke, D. R. (2006). Chronic neural recordings using silicon microelectrode arrays electrochemically deposited with a poly $(3,4-$ ethylenedioxythiophene) (PEDOT) film. J. Neural Eng. 3, 59-70.

Mannard, A., Stein, R. B., and Charles, D. (1974). Regeneration electrode units: implants for recording from single peripheral nerve fibers in freely moving animals. Science 183, 547-549.
Mattson, M. P., Haddon, R. C., and Rao, A. M. (2000). Molecular functionalization of carbon nanotubes and use as substrates for neuronal growth. J. Mol. Neurosci. 14, 175-182.

Mazzatenta, A., Giugliano, M., Campidelli, S., Gambazzi, L., Businaro, L., Markram, H., Prato, M., and Ballerini, L. (2007). Interfacing neurons with carbon nanotubes: electrical signal transfer and synaptic stimulation in cultured brain circuits. J. Neurosci. 27, 6931-6936.

Mensinger, A. F., Anderson, D. J., Buchko, C. J., Johnson, M. A., Martin, D. C., Tresco, P.A., Silver, R. B., and Highstein, S. M. (2000). Chronic recording of regenerating VIIIth nerve axons with a sieve electrode. J. Neurophysiol. 83, 611-615.

Miller, L. A., Stubblefield, K. A., Lipschutz, R. D., Lock, B. A., and Kuiken, T. A. (2008). Improved myoelectric prosthesis control using targeted reinnervation surgery: a case series. IEEE Trans. Neural Syst. Rehabil. Eng. 16, 46-50.

Navarro, X., Krueger, T. B., Lago, N., Micera, S., Stieglitz, T., and Dario, P. (2005). A critical review of interfaces with the peripheral nervous system for the control of neuroprostheses and hybrid bionic systems. J. Peripher. Nerv. Syst. 229-258.

Ni, Y., Hu, H., Malarkey, E. B., Zhao, B., Montana, V., Haddon, R. C., and Parpura, V. (2005). Chemically functionalized water soluble single-walled carbon nanotubes modulate neurite outgrowth. J. Nanosci. Nanotechnol. 5, 1707-1712.

Nicolelis, M. A. (2003). Brain-machine interfaces to restore motor function and probe neural circuits. Nat. Rev. Neurosci. 4, 417-422.

Normann, R. A. (2007). Technology insight: future neuroprosthetic therapies for disorders of the nervous system. Nat. Clin. Pract. Neurol. 3, 444-452.

Owings, M. F., and Kozak, L. J. (1998). Ambulatory and inpatient procedures in the United States, 1996. Vital Health Stat. 13, 1998, 1-119.

Panetsos, F., Avendano, C., Negredo, P., Castro, J., and Bonacasa, V. (2008). Neural prostheses: electrophysiological and histological evaluation of central nervous system alterations due to long-term implants of sieve electrodes to peripheral nerves in cats. IEEE Trans. Neural Syst. Rehabil. Eng. 16, 223-232.

Polikov, V. S., Tresco, P. A., and Reichert, W. M. (2005). Response of brain tissue to chronically implanted neural electrodes. J. Neurosci. Methods 148, 1-18.

Rejali, D., Lee, V. A., Abrashkin, K. A. Humayun, N., Swiderski, D. L., and Raphael, Y. (2007). Cochlear implants and ex vivo BDNF gene therapy protect spiral ganglion neurons. Hear Res. $228,180-187$.

Richardson, R. T., Thompson, B., Moulton, S., Newbold, C., Lum, M. G., Cameron, A., Wallace, G., Kapsa, R. Clark, G., and O’Leary, S. (2007). The effect of polypyrrole with incorporated neurotrophin-3 on the promotion of neurite outgrowth from auditory neurons. Biomaterials 28 , 513-523.

Romero, M. I., Rangappa, N., Li, L., Lightfoot, E., Garry, M. G., and Smith, G. M. (2000). Extensive sprouting of sensory afferents and hyperalgesia induced by conditional expression of nerve growth factor in the adult spinal cord. J. Neurosci. 20, 4435-4445.

Romero, M. I., Lin, L., Lush, M. E., Lei, L. Parada, L. F., and Zhu, Y. (2007) Deletion of Nfl in neurons induces increased axon collateral branching after dorsal root injury. J. Neurosci. 27, 2124-2134.

Seymour, J. P., and Kipke, D. R. (2007) Neural probe design for reduced tissue encapsulation in CNS. Biomaterials 28 3594-3607.

Vetter, R. J., Williams, J. C., Hetke, J. F., Nunamaker, E. A., and Kipke, D. R. (2004). Chronic neural recording using silicon-substrate microelectrode arrays implanted in cerebral cortex. IEEE Trans. Biomed. Eng. 51, 896-904.

Wang, K., Fishman, H. A., Dai, H., and Harris, J. S.(2006). Neural stimulation with a carbon nanotube microelectrode array. Nano Lett. 6 , 2043-2048.

Williams, J. C., Hippensteel, J. A., Dilgen, J., Shain, W., and Kipke, D. R. (2007).Complex impedance spectroscopy for monitoring tissue responses to inserted neural implants. J. Neural Eng. 4, 410-423.

Yamagata, T., Miller, J. M., Ulfendahl, M., Olivius, N. P., Altschuler, R. A., Pyykko, I., and Bredberg, G. (2004). Delayed neurotrophic treatment preserves nerve survival and electrophysiological responsiveness in neomycin-deafened guinea pigs. $J$. Neurosci. Res. 78, 75-86.

Yu, Z., McKnight, T. E., Ericson, M. N., Melechko, A. V., Simpson, M. L., and Ill, B. M. (2007). Vertically aligned carbon nanofiber arrays record electrophysiological signals from hippocampal slices. Nano Lett. 7 , 2188-2195

Conflict of Interest Statement: The authors declare that the research was conducted in the absence of any commercial or financial relationships that could be construed as a potential conflict of interest.

Received: 08 December 2008; paper pending published: 24 January 2009; accepted: 01 May 2009; published online: 26 May 2009.

Citation: Garde K, Keefer E, Botterman B, Galvan P and Romero MI (2009) Early interfaced neural activity from chronic amputated nerves. Front. Neuroeng. (2009) 2:5. doi:10.3389/neuro.16.005.2009

Copyright (C) 2009 Garde, Keefer, Botterman, Galvan and Romero. This is an open-access article subject to an exclusive license agreement between the authors and the Frontier Research Foundation, which permits unrestricted use, distribution, and reproduction in any medium, provided the original authors and source are credited. 